\title{
Human Hippocampal and Parahippocampal Theta during Goal-Directed Spatial Navigation Predicts Performance on a Virtual Morris Water Maze
}

\author{
Brian R. Cornwell, ${ }^{1}$ Linda L. Johnson, ${ }^{1}$ Tom Holroyd, ${ }^{2}$ Frederick W. Carver, ${ }^{2}$ and Christian Grillon ${ }^{1}$ \\ ${ }^{1}$ Mood and Anxiety Disorders Program, and ${ }^{2}$ Magnetoencephalography Core Facility, National Institute of Mental Health, Bethesda, Maryland 20892
}

\begin{abstract}
The hippocampus and parahippocampal cortices exhibit theta oscillations during spatial navigation in animals and humans, and in the former are thought to mediate spatial memory formation. Functional specificity of human hippocampal theta, however, is unclear. Neuromagnetic activity was recorded with a whole-head 275-channel magnetoencephalographic (MEG) system as healthy participants navigated to a hidden platform in a virtual reality Morris water maze. MEG data were analyzed for underlying oscillatory sources in the 4-8 Hz band using a spatial filtering technique (i.e., synthetic aperture magnetometry). Source analyses revealed greater theta activity in the left anterior hippocampus and parahippocampal cortices during goal-directed navigation relative to aimless movements in a sensorimotor control condition. Additional analyses showed that left anterior hippocampal activity was predominantly observed during the first one-half of training, pointing to a role for this region in early learning. Moreover, posterior hippocampal theta was highly correlated with navigation performance, with the former accounting for $76 \%$ of the variance of the latter. Our findings suggest human spatial learning is dependent on hippocampal and parahippocampal theta oscillations, extending to humans a significant body of research demonstrating such a pivotal role for hippocampal theta in animal navigation.
\end{abstract}

Key words: hippocampus; magnetoencephalography; Morris water maze; spatial memory; synthetic aperture magnetometry; theta rhythm

\section{Introduction}

There is growing consensus that hippocampal processes are involved in spatial memory and navigation in the human brain (Maguire et al., 1999; Spiers et al., 2001; Burgess et al., 2002). Intracranial electroencephalographic (iEEG) findings indicate that, as in other animals, the human hippocampus and surrounding parahippocampal cortices exhibit oscillations of local field potentials in the $\sim 4-8 \mathrm{~Hz}$ frequency range during spatial navigation, what is referred to as the theta rhythm (Kahana et al., 1999; Caplan et al., 2001, 2003; Ekstrom et al., 2005). In rodents, for instance, theta oscillations are thought to provide a critical timing mechanism for synaptic plasticity and thus are centrally implicated in processes underlying memory encoding (O'Keefe and Recce, 1993; Skaggs et al., 1996; Mehta et al., 2002; Buzsaki, 2005). A similar function of hippocampal theta in humans has not been established. Rather, evidence from iEEG studies shows that theta oscillations are elicited by movements in virtual reality (Caplan et al., 2001, 2003) and increased by environmental complexity (Kahana et al., 1999), but they are not selectively observed under conditions requiring spatial information to be encoded or retrieved (Ekstrom et al., 2005).

Received Aug. 21, 2007; revised April 1, 2008; accepted April 21, 2008.

This work was supported by the intramural research program at the National Institute of Mental Health.

Correspondence should be addressed to Brian R. Cornwell, Mood and Anxiety Disorders Program, National Institute of Mental Health, 15K North Drive, MSC 2670, Bethesda, MD 20892. E-mail: cornwellb@mail.nih.gov.

D01:10.1523/JNEUROSCI.5001-07.2008

Copyright $\odot 2008$ Society for Neuroscience $\quad$ 0270-6474/08/285983-08\$15.00/0
We administered to healthy participants a virtual reality analog of the Morris water maze task (vMWM) used extensively to study spatial navigation and learning in rodents (Morris, 1981; Morris et al., 1982). Similar in task structure to the actual version, participants navigate by joystick to a hidden, submerged platform in a virtual circular pool of water (Fig. 1). Optimal navigation to the hidden platform from different starting locations requires formation of a cognitive map from distal visual cues on the surrounding walls (Jacobs et al., 1998a,b); learning can be readily inferred by quantifying performance in terms of path length or latency to reach the platform across trials. Studies with this virtual task have established gender-, age-, and stress-related performance changes in healthy participants (Astur et al., 1998; Driscoll et al., 2003; Duncko et al., 2007). Driscoll et al. (2003) further reported that hippocampal volume was correlated with performance in an aging population, and Astur et al. (2002) demonstrated resection of either left or right hippocampus was accompanied by significant performance deficits, suggesting vMWM performance is hippocampal dependent.

Based on iEEG findings, we predicted hippocampal and parahippocampal cortical regions would exhibit theta oscillations during goal-directed navigation in the vMWM. To noninvasively measure theta oscillations as participants navigated in the virtual pool, magnetoencephalographic (MEG) recordings were made with a whole-head 275-channel magnetometer and subjected to source analysis by spatial filtering [synthetic aperture magnetometry (SAM)] (Robinson and Vrba, 1999). A spatial filter can iso- 


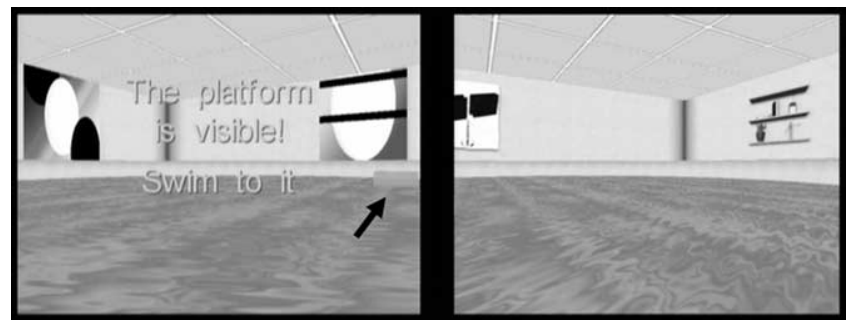

Figure 1. Snapshots of the virtual Morris water maze environments that were presented in color to participants. In one pool (left), distal visual cues on the surrounding walls were abstract patterns and in the other (right) they were concrete objects (e.g., shelves). Note that, in the goal-directed navigation condition, the platform was hidden for $15 \mathrm{~s}$, after which it became visible (indicated by the arrow) if a participant failed to find it.

late oscillatory signals in a specific frequency band at any given point in the brain by exploiting the statistical relationships of signal measurements between sensors. Multiple spatial filters are constructed sequentially to create a volumetric statistical image representing the power distribution of a given oscillatory signal throughout the brain (Hillebrand et al., 2005). Here, we mapped oscillatory changes in the $4-8 \mathrm{~Hz}$ theta band across the brain during goal-directed navigation and during aimless movements (control condition).

\section{Materials and Methods}

Participants. Fifteen healthy participants (seven women, right hand dominant) were recruited for participation (mean age, 26 years; range, 19-39 years) and completed both the MEG testing session and a separate session to obtain an anatomical magnetic resonance image (MRI) for source analysis. Two additional subjects who completed both sessions were excluded from analysis because of either excessive head movement or significant physiological artifacts. This study was approved by the National Institute of Mental Health Institutional Review Board, and all participants gave informed consent before participation. Participant inclusion criteria included the following: (1) no past or current psychiatric disorders as per Structured Clinical Interview for DSM-IV (First et al., 1995), (2) no current use of psychoactive medications as per self-report, and (3) no current use of illicit drugs determined by urine analysis. Participants were also screened for metallic implants, tattoos, and other nonremovable metallic objects.

Virtual Morris water maze. Participants completed 40 trials in which they moved with a fiber optic joystick around a virtual circular pool presented with commercially available software (Fig. 1) (NeuroInvestigations), back-projected onto a screen at a distance of $\sim 75 \mathrm{~cm}$. Participants began each trial facing the edge of the pool at one of four starting locations. On one-half of the trials, participants were instructed to navigate as quickly as possible to a hidden platform in a fixed location (navigation condition). If the hidden platform was not found within $15 \mathrm{~s}$, it became visible and participants were instructed to navigate to it to finish the trial. On the other one-half of the trials, participants moved aimlessly for the $15 \mathrm{~s}$ duration of each trial (sensorimotor control condition). Participants were instructed on these trials to move around the pool continuously until the trial ended. Their behavior was closely observed on a computer monitor outside the shielded room. We confirmed that participants did not stop moving for $>1-2 \mathrm{~s}$ at a time and redirected their paths when they reached the edge of the pool, suggesting that they were attending to the visual display during this condition. To limit retrieval of spatial knowledge related to the hidden platform location on control trials, separate virtual pool environments were used for the two conditions, with the only difference being the distal cues on the walls (concrete objects such as a door and shelves in one environment vs abstract patterns in the other) (Fig. 1).

Navigation and sensorimotor control trials were alternated in pairs (i.e., two navigation, two control, two navigation trials, etc.), and participants used the cues on the walls, after turning around from the edge of the pool, to determine at the start of each trial whether they needed to search for the platform or move aimlessly. There were $5 \mathrm{~s}$ breaks between trials, during which no visual stimulus was presented. Participants re ceived two exposures to each pool environment before the MEG recording, to familiarize them with the cues and to practice using the joystick. The pool environment containing the hidden platform was counterbalanced across participants. Total path lengths and latencies to find the platform were automatically recorded by the program, trial-by-trial, and stored for later analysis of behavioral performance.

Data acquisition. Neuromagnetic activity was measured with a CTFOMEGA 275-channel whole-head magnetometer (VSM MedTech) in a magnetically shielded room (Vacuumschmelze) using synthetic third gradient balancing for active noise cancellation. Magnetic fields were digitized at a $1200 \mathrm{~Hz}$ rate with a bandwidth of $0-300 \mathrm{~Hz}$. Fiducial coils were attached to the nasion and right and left preauricular sites. Coils were energized before and after data collection to determine head position in the dewar for coregistration with the anatomical MRI. Total head displacement during the run was recorded at the end.

MRIs were obtained in a separate session using a 3 tesla whole-body scanner (GE Signa). A high-resolution T1-weighted anatomical image was obtained from each participant with the following parameters: field of view, 24, $1.2 \mathrm{~mm}$ axial slices; matrix, $256 \times 256 \times 128$. The same three fiducial sites in which electrical coils had been attached during the MEG session were marked in the MRI with radiological markers (IZI Medical Products).

Synthetic aperture magnetometry. SAM is a minimum variance beamformer algorithm for estimating power changes within specific frequency bands and time windows across the whole brain with no a priori assumptions about the number of active sources or their spatial locations (Robinson and Vrba, 1999; Vrba and Robinson, 1999; Hillebrand et al., 2005). A high degree of correspondence between SAM source activations and functional MRI (fMRI)-blood oxygen level-dependent activations has been demonstrated within the same experimental paradigms (Singh et al., 2002; Schultz et al., 2004). The primary advantage of SAM over other MEG/EEG source analysis techniques is that the former performs optimally on unaveraged epochs of data, and therefore is better equipped to identify sources that are not phase-locked to a stimulus (i.e., induced oscillations). In the present case, hippocampal theta is very unlikely to show a consistent phase relationship across trials given the variability in when participants begin navigating or processing spatial information.

In general, source space is parsed into a three-dimensional grid with a unique beamformer or spatial filter constructed at each point from the covariance matrix of sensor data within a defined time-frequency window and optimized to pass activity only at that point and suppress activity elsewhere. This is done by minimizing the output of the spatial filter subject to the constraint of unity gain for a current source at the target location, which is obtained by calculating the biomagnetic forward solution. Because the beamformer passes both the signal of interest and noise it is important to either estimate noise amplitudes and normalize beamformer outputs accordingly (i.e., single-state imaging) or contrast active and control states and implicitly control for noise (i.e., dual-state imaging) under the assumption that the two states have similar noise levels (for additional details, see Hillebrand et al., 2005). We chose the latter method.

Covariance matrices were computed from 20 unaveraged $1 \mathrm{~s}$ epochs of the navigation condition after applying a 4-8 Hz bandpass filter. Dualstate images were constructed by normalizing the $1 \mathrm{~s}$ active windows recorded during navigation versus the $1 \mathrm{~s}$ control windows recorded during the pretrial baseline period. By creating dual-state images, we controlled for pretrial baseline activity and were able to quantify the degree to which theta power increased during navigation. Source space was sampled in $5 \mathrm{~mm}$ steps with equivalent current dipole sources at each location. Magnetic fields were modeled using a multisphere head model derived from each participant's gross brain morphology. Active windows were advanced in $250 \mathrm{~ms}$ increments (or one lower bound theta cycle) from the beginning of the trial up to $5.25 \mathrm{~s}$ to capture source activity across the majority of the navigation period (5.25 s was the upper limit determined by the fastest time to the platform by a participant). Thus, the 
sliding window analysis produced 18 different source volumes. These same steps were then repeated for the sensorimotor control condition.

Group statistical analysis. Using AFNI (Cox, 1996), individual SAM volumes for each condition were spatially warped to a Talairach brain template to allow for group analysis in a standardized source space and within-volume normalized using a $z$-score transformation. The latter procedure was performed to limit the influence of global power on local power in a particular brain region. Paired $t$ tests were conducted for voxelwise group analysis, comparing source power between the navigation and sensorimotor control conditions for each time window. Because participants moved around the pool during each condition, this comparison was intended to reveal neural activity related to the additional spatial cognitive processes mediating navigation to the hidden platform. Given our a priori hypothesis that the hippocampus and surrounding parahippocampal cortices will be major sources of theta during navigation, we performed a small-volume correction using a bilateral medial temporal cortical region of interest (ROI) ( $\sim 820$ voxels) based on the automated Talairach Daemon (Lancaster et al., 2000). Within this ROI across all 18 time windows, we used a statistical threshold that yielded a false discovery rate of $<5 \%(q<0.05)$ (Genovese et al., 2002).

Secondary analyses were also performed on delta, alpha, beta, and gamma bands to determine the specificity of theta activity observed. All SAM procedures were the same as above except that for delta, alpha, beta, and gamma source analyses, bandpass filters of $1-4,8-15,15-30$, and $30-50 \mathrm{~Hz}$ were applied before covariance matrices were computed, respectively.

To determine whether hippocampal/parahippocampal theta was related to behavioral performance on the vMWM, difference volumes (navigation pseudo- $F$ volume minus control pseudo- $F$ volume) were computed for each participant and subjected to correlation analysis with the criterion variable being average path length taken to the platform or average latency to reach the platform. Nonparametric Spearman correlation coefficients were generated at each voxel location and local minima were identified for each time window using an uncorrected threshold of $p<0.001$. Only local minima were relevant to our goal in that they reflected regions in which there was an association between differentially greater theta in the navigation condition and shorter path lengths to the platform or shorter latencies to reach the platform (i.e., superior performance or more efficient navigation).

\section{Results}

\section{Navigation performance}

To demonstrate spatial learning in the vMWM task, two dependent variables were used to assess changes in navigation performance across five blocks of trials (four trials per block) with a hidden platform: (1) path length taken from the starting position to the hidden platform and (2) total latency to reach the platform. Within-subject ANOVAs showed that path length and latency to find the platform were significantly different across blocks: $F_{(4,40.62)}=2.87$, mean square error $(\mathrm{MSE})=0.05, p=0.05, \eta^{2}=$ 0.17 , Huynh-Feldt $\varepsilon=0.73$, and $F_{(4,39.79)}=6.26$, MSE $=8.53$, $p<0.005, \eta^{2}=0.31$, Huynh-Feldt $\varepsilon=0.71$, respectively. Participants showed an overall decrease in latency and path length to the platform over the training session (Fig. 2).

\section{Localization of navigation-related theta activity}

Before presenting results of source analyses, we conducted timefrequency analyses of raw sensor data using the Stockwell transform (Stockwell et al., 1996). Sensors over left and right temporal cortices were searched iteratively, because it was not clear which sensors might be optimally positioned to measure activity from medial temporal cortical sources given their depth (and also given the variability in participants' head positions inside the dewar). We found three sensors positioned approximately over left temporal cortical regions that captured a distinct increase in power, on average across participants, centered $\sim 5 \mathrm{~Hz}$ and $2 \mathrm{~s}$

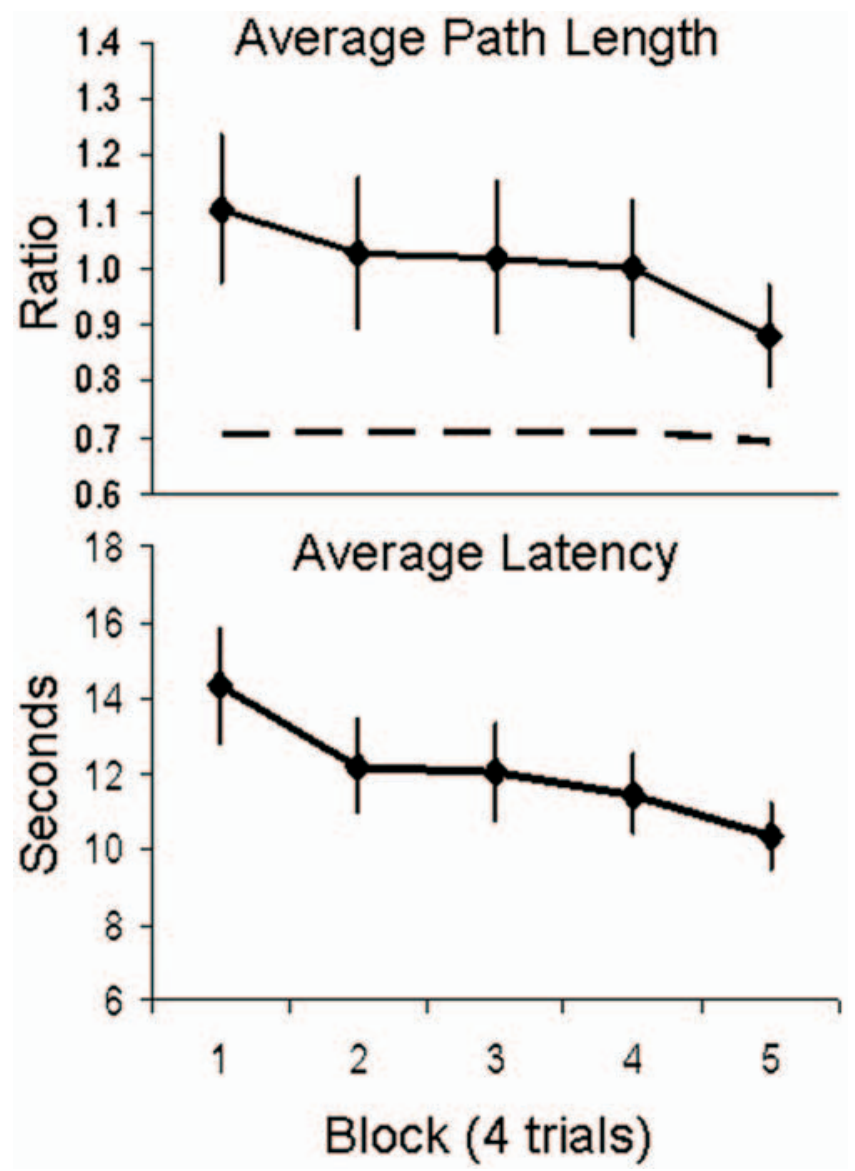

Figure 2. Spatial learning curves during MEG data acquisition. Navigation performance is quantified by path length taken from the starting position to the hidden platform (top) and total latency to reach the platform over five blocks of four trials (bottom). Path length is expressed relative to the diameter of the pool. The dashed line represents the ideal path length relative to the diameter of the pool, namely, a straight line from the various starting positions to the platform. Error bars represent SEMs.

relative to trial onset when participants searched for the platform (Fig. 3). These same sensors captured less of a power increase in the theta frequency band during aimless movements. Increased theta power was less discernible in time-frequency analyses of other sensor data for both conditions.

For the main hypothesis that the hippocampus and parahippocampal cortices will exhibit theta oscillations during goaldirected navigation relative to aimless movements in the virtual pool (sensorimotor control condition), and which might be contributing to the theta activity recorded by the left temporal sensors, spatial filtering analyses (i.e., SAM) were conducted in $1 \mathrm{~s}$ windows using a bandpass filter of $4-8 \mathrm{~Hz}$. Source volumes for each condition and latency window (relative to trial onset) were directly contrasted using paired $t$ tests, voxel-by-voxel within our defined medial temporal cortex ROI. We identified two local maxima of differential theta power (Fig. 4). First, theta peaked in the anterior portion of the left hippocampus 1-2 s after trial onset. Second, theta peaked slightly anterior and inferior to the left hippocampus, in parahippocampal cortex, $\sim 500 \mathrm{~ms}$ after the left hippocampal activation or 1.5-2.5 s after trial onset. Both sources were driven by greater theta synchronization from baseline during goal-directed navigation relative to aimless movements, as opposed to greater desynchronization in the latter condition compared with the former. Time courses of source activity suggested that theta synchronization was comparable between 
the two conditions at the start of the trial, and that only during goal-directed navigation did theta show sustained synchronization across the first few seconds (Fig. 4). A third peak of theta activity was identified in the left uncus/parahippocampal cortex (BA 36, -30, -4, -31 mm) 4-5 s after trial onset, but it appeared to be driven equally by increased power during goaldirected navigation and decreased power during aimless movements.

Whole-brain analyses were also conducted using a less conservative statistical criterion for exploratory purposes. Table 1 presents those regions showing greater theta activity during goal-directed navigation relative to aimless swimming at $p<$ 0.001 (uncorrected). The overall pattern suggests that regions exhibiting theta activity were predominantly confined to lateral prefrontal and temporal cortices in the left hemisphere.

Localization of other oscillatory changes We performed secondary analyses in the other major frequency bands [delta (1-4 $\mathrm{Hz})$, alpha $(8-15 \mathrm{~Hz})$, beta $(15-30 \mathrm{~Hz})$, and gamma bands $(30-50 \mathrm{~Hz})]$ and used a similar small-volume multiplecomparison correction approach for statistical analysis to determine whether hippocampal and parahippocampal regions exhibited oscillatory power increases other than theta during goal-directed navigation. No evidence for increased power in these bands in hippocampal and parahippocampal structures was obtained.

Peak differential power increases were extracted for each frequency band independent of time and location in the medial temporal cortices to directly test whether this region showed theta activity exclusively throughout goal-directed navigation. A 2 (condition) $\times 5$ (frequency band) repeated-measures ANOVA revealed a main effect of condition $\left(F_{(1,14)}=36.70 ; \mathrm{MSE}=1.19 ; p<0.001 ; \eta^{2}=0.72\right)$, but no main effect for frequency band $\left(F_{(2.14,29.96)}=1.31\right.$; MSE $=$ 1.42 , NS; Huynh-Feldt $\varepsilon=0.54)$, nor condition by frequency band interaction $(F<1)$. All major frequency bands, as opposed to only theta, tended to show increased power at various times during goal-directed navigation relative to aimless swimming. Effect size for the maximal theta source (Cohen's $d=1.66$ ) was larger than for maximal sources of other bands: delta $(d=1.08)$, alpha $(d=1.19)$, beta $(d=1.15)$, and gamma $(d=1.26)$. The failure to show significant activations in other frequency bands in the previous analyses, thus, may be the result of insufficient statistical power given the differences in effect sizes.

\section{Correlation between hippocampal theta and navigation performance}

Nonparametric Spearman correlations were conducted between normalized pseudo- $F$ difference volumes (navigation minus control) and navigation performance to determine whether medial temporal cortical structures showed differential greater theta during the navigation condition that was linearly associated with navigation performance (average path length and average latency) across participants. With respect to average path length, a local minimum (i.e., peak negative correlation coefficients) was identified in the left posterior hippocampus/parahippocampal region between 0.25 and $1.25 \mathrm{~s}(-24,-37,0 \mathrm{~mm})$ (Fig. 5), suggesting that differentially greater theta here was associated with better navigation performance $r_{(13)}=-0.87, p<0.001$. With respect to average latency, no medial temporal cortical structures showed negatively correlated activity that reached our statistical threshold.

We sought to determine whether left posterior hippocampal activity covaried with path length within each participant on a trial by trial basis. Single-trial estimates of theta power were calculated at the location showing maximal correlation between average theta power and average path length across participants in the relevant time window (i.e., $0.25-1.25 \mathrm{~s}$ ). These estimates were then normalized to pretrial baseline power estimates to compute a similar ratio value as in the main analyses; we then subtracted out a similar ratio derived from single-trial power estimates obtained for the sensorimotor control condition. To control for variability in starting distance from the platform across trials, observed path length was divided by ideal path length (i.e., straight line from starting position to platform location). Nonparametric Spearman correlations showed 1 of 15 participants 


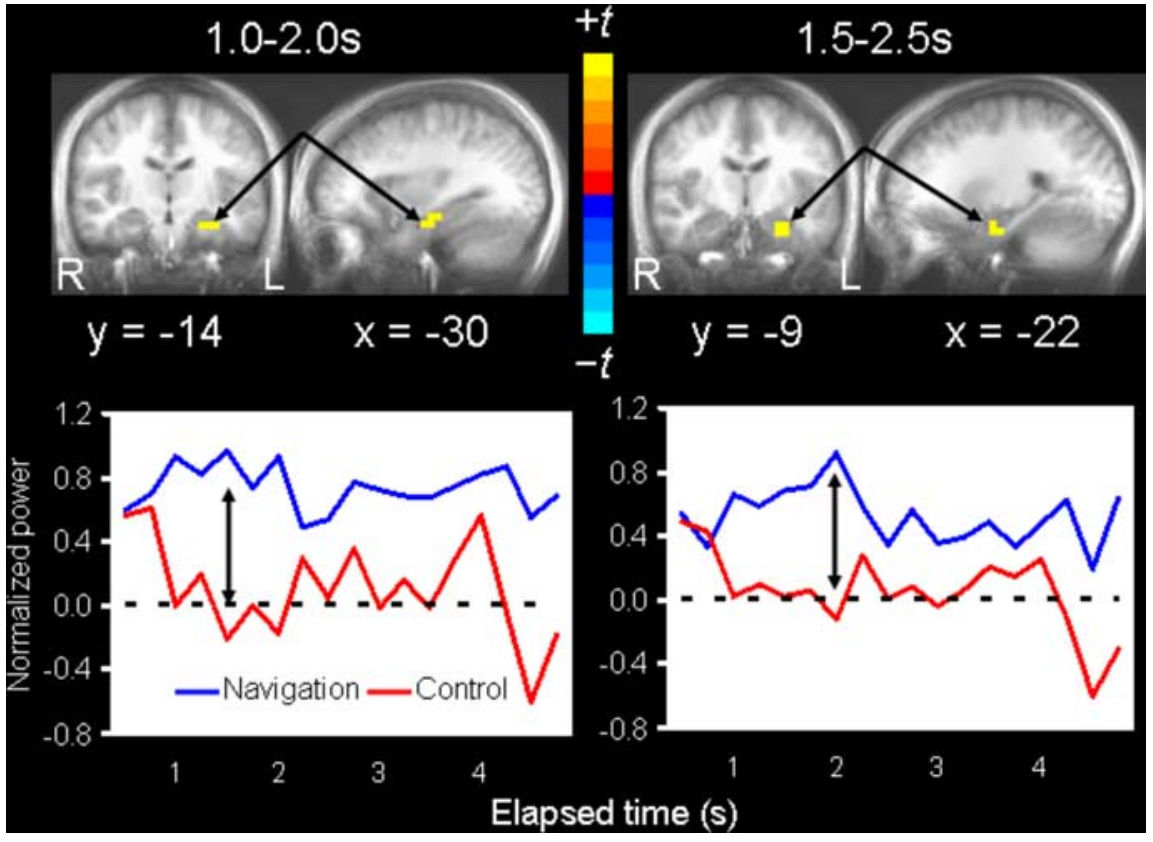

Figure 4. Regional theta activity related to goal-directed navigation. Coronal and sagittal views depicting medial temporal regions with greater theta activity during goal-directed navigation relative to aimless movements based on paired $t$ tests, thresholded at $p=0.00011$ (false discovery rate, $<5 \%$ ). Statistical maps are overlaid on an averaged anatomical MRI and presented in radiological view (left side of the image corresponds to the right hemisphere, and vice versa). Top left, Left hippocampal activation (local maximum, $-30,-14,-18 \mathrm{~mm}$ in Talairach space) was observed $1-2 \mathrm{~s}$ after trial onset. Top right, Left hippocampal/parahippocampal activation (local maximum, $-22,-9,-21 \mathrm{~mm}$ ) was also observed $1.5-2.5 \mathrm{~s}$ after trial onset. The line graphs below source images show the average change in normalized pseudo- $F$ power ratios (i.e., power relative to pretrial baselines) for each local maximum from the start of the trial through $5.25 \mathrm{~s}$ for both the navigation and sensorimotor control conditions. Each time point represents power integrated over a $1 \mathrm{~s}$ window and is labeled along the $x$-axis according to its midpoint.

for which a significant negative correlation between posterior hippocampal theta and path length was evident $\left(r_{(18)}=-0.57\right.$; $p<0.01)$. The average within-participant correlation coefficient was -0.06 .

\section{Medial temporal theta in early versus late training}

To investigate whether hippocampal and parahippocampal sources exhibited differential theta activity during early versus later training, we conducted a set of source analyses in the two time windows showing significant hippocampal and parahippocampal theta activity (i.e., $1-2$ and $1.5-2.5 \mathrm{~s}$ after trial onset). Covariance matrices were computed and source power was estimated in the same way as in the main analyses but for the first 10 trials and last 10 trials separately. In the $1.5-2.5 \mathrm{~s}$ window, we found a source in the left anterior hippocampus $(-33,-17,-12$ $\mathrm{mm}$ ) showing a significant condition by phase (early vs late training) interaction at $p<0.05$ (uncorrected), which was driven by greater differential activity early in training, $p<0.001$ (uncorrected), but not later in training (mean $\pm \mathrm{SD}, 0.64 \pm 0.96$ pseudo- $F$ for the navigation condition vs $-0.19 \pm 0.77$ pseudo- $F$ for the control condition in early training, and $-0.28 \pm 0.99$ vs $0.14 \pm 1.1$ in late training).

\section{Lateralization of theta in medial temporal cortices}

We also directly tested whether sources exhibiting increased theta activity in the left medial temporal cortices showed differentially greater theta activity during goal-directed navigation (compared with aimless movements) than sources in the right medial temporal cortices. This was done for local maxima in left and right medial temporal cortices independent of time and location. The local maximum in the right medial temporal cortices was observed between 1 and $2 \mathrm{~s}$ after trial onset in parahippocampal cortex $(23,-32,-11 \mathrm{~ms})$. A 2 (condition) $\times 2$ (side) repeated-measures ANOVA revealed a significant main effect of condition $\left(F_{(1,14)}=15.86 ;\right.$ MSE $=0.32 ; p<$ $\left.0.001 ; \eta^{2}=0.78\right)$ and a marginally significant main effect of side $\left(F_{(1,14)}=3.37\right.$; MSE $\left.=0.26 ; p<0.10 ; \eta^{2}=0.19\right)$, but no interaction $(F<1)$. Thus, there was no evidence indicating a strict lateralization of theta activity to left medial temporal cortices during goal-directed navigation.

\section{Discussion}

In agreement with iEEG findings (Kahana et al., 1999; Caplan et al., 2001, 2003; Ekstrom et al., 2005), we observed increased power in the $4-8 \mathrm{~Hz}$ theta band in the human hippocampus and surrounding parahippocampal structures in a similar behavioral context as animals, namely goaldirected navigation. Using a spatial filtering technique for source analysis, we measured the distribution of theta activity across the brain during navigation in a vMWM and also during a sensorimotor control condition (i.e., aimless movements). Two early peaks of left hippocampal and parahippocampal theta activity were observed on trials in which participants navigated to the fixed location of the hidden platform relative to trials in which they moved aimlessly around the virtual pool (Fig. 4). An additional source of increased theta power was localized to left parahippocampal cortices later in the trial period (i.e., 3.75-4.75 s relative to trial onset). These results suggest that hippocampal and parahippocampal theta oscillations increased specifically under conditions in which spatial information was being encoded and/or retrieved rather than being elicited solely by virtual movements in general. Although theta was the only frequency band showing significant increase in medial temporal cortices during goal-directed navigation relative to aimless movements, this power increase was not significantly greater than power increases in other bands at other time intervals. Hippocampal neuronal populations have been shown to exhibit $1-4 \mathrm{~Hz}$ oscillations during virtual navigation and may serve a similar function as $4-8 \mathrm{~Hz}$ oscillations in humans (de Araujo et al., 2002; Jacobs et al., 2007). That we could not demonstrate medial temporal sources displaying significant power changes in other bands during navigation, perhaps because of insufficient statistical power, necessitates additional investigation to clarify potential roles of other oscillations in the vMWM.

We found compelling evidence of a linear relationship between hippocampal and parahippocampal theta responses and navigation performance on the vMWM, consistent with the interpretation that oscillatory activity is involved in spatial learning. Indeed, participants exhibiting greater differential activity in the left posterior hippocampus at the start of navigation (relative to moving aimlessly) were the best performers in terms of average path length taken to the platform (Fig. 5). In other words, early hippocampal activity predicted navigation performance, with the 
former accounting for a sizeable proportion of variance in the latter (i.e., 76\%). Previous fMRI studies also found positive linear associations (i.e., $r^{2}=0.55$ and 0.31 ) between posterior hippocampal activation and virtual navigation performance (Maguire et al., 1998; Hartley et al., 2003). That we obtained a larger correlation than these previous results could be attributable to the ability of MEG to capture neural dynamics more directly than fMRI and isolate oscillatory changes in the relevant frequency bands. However, we were unable to show this same correlation at the single-trial level in more than one participant. This is likely the result of significant noise in single-trial estimates of power made at the depth of the hippocampus and perhaps reflects limitations of noninvasive MEG recordings, but nevertheless may be observed with a greater number of trials.

The robust correlation between posterior hippocampal theta and average path length emerged in the first second of navigation and may be indicative of rapid engagement of the hippocampus and parahippocampal cortices in the best navigators. This relationship may reflect a direct connection between posterior hippocampal theta activity and cognitive aspects of navigation performance or could be mediated by motor-related variables (i.e., speed). Although the relationship was obtained by factoring out theta activity during the sensorimotor control condition (i.e., by correlating difference volumes with path length), motor-related processes may not have been sufficiently controlled across these two conditions to disambiguate the meaning of the observed relationship. This problem remains unresolved in the rodent literature with some studies that have controlled for motor activity reporting cognitive-related hippocampal theta oscillations (Olvera-Cortes et al., 2004) and others not (Kelemen et al., 2005). By demonstrating similarities in hippocampal oscillatory activity across species, the current results may offer novel approaches to addressing its cognitive significance in humans.

Anterior hippocampal and parahippocampal activity was greater, in general, during goal-directed navigation relative to aimless movements, an outcome that may appear inconsistent with human studies indicating that posterior medial temporal cortical regions are especially critical to spatial navigation (Ghaem et al., 1997; Bohbot et al., 1998; Maguire et al., 1998). An important difference between previous human neuroimaging studies and the current study is that the former studies usually involved significant training outside the scanner before neural activity was measured and the latter did not. We measured neuromagnetic activity during a single session in which participants, in general, showed significant improvement in performance across trials (Fig. 2), suggesting that they were continually learn- ing and encoding the spatial environment. That the left anterior hippocampal theta activity observed was related to early encoding is supported by subsequent analyses that demonstrated that activity in this structure was clearly evident during the first 10 trials, when most participants had not mastered the task, but not the last 10, when some were approaching ceiling level performance. Wolbers and Buchel (2005), using fMRI, made a similar observation of early involvement of the left anterior hippocampus in virtual navigation when training was distributed across multiple sessions. Moreover, based on the correlations we observed in posterior hippocampal and parahippocampal regions, it could be expected that had we trained participants to a specific criterion before measuring neuromagnetic activity, we would have observed, similar to other studies, a more uniform response 
in posterior regions across participants. Nevertheless, no consensual view on functional subdivisions of the human hippocampus has emerged (Squire et al., 2004); thus, anterior hippocampal and parahippocampal oscillations during spatial learning could be involved in early encoding and in generating novel paths (Hartley et al., 2003), and/or could reflect greater attentional orienting to the pool environment containing the hidden platform (Vinogradova, 2001). More extensive training on the vMWM across multiple sessions would be necessary to clarify the significance of anterior versus posterior hippocampal activity.

Previous results point to a more critical role for the right hippocampus over the left in spatial memory processes in general (cf. Burgess et al., 2002), but this has not always been demonstrated (Astur et al., 2002; Ohnishi et al., 2006) and the left anterior hippocampus may nonetheless mediate specific component processes of spatial navigation such as binding an object, in this case the platform, to its spatial location (Mitchell et al., 2000; Kessels et al., 2004). Although the peak response in left medial temporal cortices observed here was not significantly greater in magnitude than that observed on the right during goal-directed navigation, the relative lack of evidence for right hippocampal and parahippocampal activity in the present study may be attributable to the strategies used by participants to locate the platform. That is, associative strategies that rely on only a subset of cues may have been used instead of allocentric representations (i.e., cognitive maps) of the environment that are thought to be mediated by the right hippocampus (Burgess et al., 2002). The latter is the optimal strategy when navigating from variable starting positions in the vMWM; yet simpler associative strategies seem to be sufficient to perform adequately (e.g., knowing only that the platform is left of the window). Aspects of the current procedures may have influenced the strategies used, such as the interleaved presentation of navigation and control trials that may have hindered formation of cognitive maps. Future studies could address the question of lateralization of hippocampal activity on the vMWM by more systematic manipulation of training variables and detailed characterizations of the paths taken by participants to the hidden platform.

Based on animal studies, theta is thought to behave as an order parameter by integrating sequentially activated cell assemblies into higher-order dynamical structures embodying temporal context (Buzsaki, 2005). The formation of spatial memories, and perhaps more generally episodic memories, may therefore be critically dependent on hippocampal theta oscillations (Buzsaki, 2005; Samsonovich and Ascoli, 2005). Along these lines, there is ample evidence in healthy humans that theta recorded noninvasively at the scalp (EEG) or externally (MEG) is related to memory processes such as successful encoding of target items (Klimesch et al., 1996, 1997; Sederberg et al., 2003) and maintenance of items in working memory (Gevins et al., 1997; Tesche and Karhu, 2000; Jensen and Tesche, 2002). However, few studies have specifically implicated the hippocampus or parahippocampal cortices as the primary sources of theta related to memory processes (for exceptions, see Tesche and Karhu, 2000; de Araujo et al., 2002). The current study bridges this gap between animal and human research by demonstrating that major sources of theta activity during human spatial learning are located in the hippocampus and parahippocampal cortices, and that spatial navigation performance is highly associated with theta activity in these structures. Additional research may reveal the full extent to which hippocampal theta oscillations, and other oscillatory changes, are critical to human learning and memory, spatial as well as nonspatial kinds.

\section{References}

Astur RS, Ortiz ML, Sutherland RJ (1998) A characterization of performance by men and women in a virtual Morris water task: a large and reliable sex difference. Behav Brain Res 93:185-190.

Astur RS, Taylor LB, Mamelak AN, Philpott L, Sutherland RJ (2002) Humans with hippocampus damage display severe spatial memory impairments in a virtual Morris water task. Behav Brain Res 132:77-84.

Bohbot VD, Kalina M, Stephankova K, Spackova N, Petrides M, Nadel L (1998) Spatial memory deficits in patients with lesions to the right hippocampus and to the right parahippocampal cortex. Neuropsychologia 36:1217-1238.

Burgess N, Maguire EA, O'Keefe J (2002) The human hippocampus and spatial and episodic memory. Neuron 35:625-641.

Buzsaki G (2005) Theta rhythm of navigation: link between path integration and landmark navigation, episodic and semantic memory. Hippocampus 15:827-840.

Caplan JB, Madsen JR, Raghavachari S, Kahana MJ (2001) Distinct patterns of brain oscillations underlie two basic parameters of human maze learning. J Neurophysiol 86:368-380.

Caplan JB, Madsen JR, Schulze-Bonhage A, Aschenbrenner-Scheibe R, Kahana MJ (2003) Human theta oscillations related to sensorimotor integration and spatial learning. J Neurosci 23:4726-4736.

Cox RW (1996) AFNI: software for analysis and visualization of functional magnetic resonance neuroimages. Comput Biomed Res 29:162-173.

de Araujo DB, Baffa O, Wakai RT (2002) Theta oscillations and human navigation: a magnetoencephalography study. J Cogn Neurosci 14:70-78.

Driscoll I, Hamilton DA, Petropoulos H, Yeo RA, Brooks WM, Baumgartner RN, Sutherland RJ (2003) The aging hippocampus: cognitive, biochemical and structural findings. Cereb Cortex 13:1344-1351.

Duncko R, Cornwell B, Cui L, Merikangas KR, Grillon C (2007) Acute exposure to stress improves performance in trace eyeblink conditioning and spatial learning tasks in healthy men. Learn Mem 14:329-335.

Ekstrom AD, Caplan JB, Ho E, Shattuck K, Fried I, Kahana MJ (2005) Human hippocampal theta activity during virtual navigation. Hippocampus 15:881-889.

First MB, Spitzer RI, Williams JBW, Gibbon M (1995) Structured clinical interview fro DSM-IV (SCID). Washington, DC: American Psychiatric Association.

Genovese CR, Lazar NA, Nichols T (2002) Thresholding of statistical maps in functional neuroimaging using false discovery rates. NeuroImage 15:772-786.

Gevins A, Smith ME, McEvoy L, Yu D (1997) High-resolution EEG mapping of cortical activation related to working memory: effects of task difficulty, type of processing, and practice. Cereb Cortex 7:374-385.

Ghaem O, Mellet E, Crivello F, Tzourio N, Mazoyer B, Berthoz A, Denis M (1997) Mental navigation along memorized routes activates the hippocampus, precuneus, and insula. NeuroReport 8:739-744.

Hartley T, Maguire EA, Spiers HJ, Burgess N (2003) The well-worn route and the path less traveled: distinct neural bases of route following and wayfinding in humans. Neuron 37:877-888.

Hillebrand A, Singh KD, Holliday IE, Furlong PL, Barnes GR (2005) A new approach to neuroimaging with magnetoencephalography. Hum Brain Mapp 25:199-211.

Jacobs J, Kahana MJ, Ekstrom AD, Fried I (2007) Brain oscillations control timing of single-neuron activity in humans. J Neurosci 27:3839-3844.

Jacobs WJ, Laurance HE, Thomas KGF (1998a) Place learning in virtual space. I. Acquisition, overshadowing, and transfer. Learn Motiv 28:521-541.

Jacobs WJ, Thomas KGF, Laurance HE, Nadel L (1998b) Place learning in virtual space. II. Topographical relations as one dimension of stimulus control. Learn Motiv 29:288-308.

Jensen O, Tesche CD (2002) Frontal theta activity in humans increases with memory load in a working memory task. Eur J Neurosci 15:1395-1399.

Kahana MJ, Sekuler R, Caplan JB, Kirschen M, Madsen JR (1999) Human theta oscillations exhibit task dependence during virtual maze navigation. Nature 399:781-784.

Kelemen E, Moron I, Fenton AA (2005) Is the hippocampus theta rhythm related to cognition in a non-locomotor place recognition task? Hippocampus 15:472-479.

Kessels RPC, Hendriks MPH, Schouten J, van Asselen M, Postma A (2004) Spatial memory deficits in patients after unilateral selective amygdalohippocampectomy. J Int Neuropsychol Soc 10:907-912. 
Klimesch W, Doppelmayr M, Russegger H, Pachinger T (1996) Theta band power in the human scalp EEG and the encoding of new information. NeuroReport 7:1235-1240.

Klimesch W, Doppelmayr M, Schimke H, Ripper B (1997) Theta synchronization and alpha desynchronization in a memory task. Psychophysiology 34:169-176.

Lancaster JL, Woldorff MG, Parsons LM, Liotti M, Freitas CS, Rainey L, Kochunov PV, Nickerson D, Mikiten SA, Fox PT (2000) Automated Talairach atlas labels for functional brain mapping. Hum Brain Mapp 10:120-131

Maguire EA, Burgess N, Donnett JG, Frackowiak RSJ, Frith CD, O'Keefe J (1998) Knowing where, and getting there: a human navigation network. Science 280:921-924.

Maguire EA, Burgess N, O'Keefe J (1999) Human spatial navigation: cognitive maps, sexual dimorphism, and neural substrates. Cur Opin Neurobiol 9:171-177.

Mehta MR, Lee AK, Wilson MA (2002) Role of experience and oscillations in transforming a rate code into a temporal code. Nature 417:741-746.

Mitchell KJ, Johnson MK, Raye CL, D’Esposito M (2000) fMRI evidence of age-related hippocampal dysfunction in feature binding in working memory. Cogn Brain Res 10:197-206.

Morris RGM (1981) Spatial localization does not require the presence of local cues. Learn Motiv 12:239-260.

Morris RGM, Garrud P, Rawlins JN, O'Keefe J (1982) Place navigation impaired in rats with hippocampal lesions. Nature 297:681-683.

Ohnishi T, Matsuda H, Hirakata M, Ugawa Y (2006) Navigation ability dependent neural activation in the human brain: an fMRI study. Neurosci Res 55:361-369.

O'Keefe J, Recce ML (1993) Phase relationship between hippocampal place units and the EEG theta rhythm. Hippocampus 3:317-330.

Olvera-Cortes E, Guevara MA, Gonzalez-Burgos I (2004) Increase of the hippocampal theta activity in the Morris water maze reflects learning rather than motor activity. Brain Res Bull 62:379-384.

Robinson SE, Vrba J (1999) Functional neuroimaging by synthetic aperture magnetometry (SAM). In: Recent advances in biomagnetism (Yoshimoto
T, Kotani M, Kuriki S, Karibe, H, Nakasato N, eds), pp 302-305. Sendai, Japan: Tohoku UP.

Samsonovich AV, Ascoli GA (2005) A simple neural network model of the hippocampus suggesting its pathfinding role in episodic memory retrieval. Learn Mem 12:193-208.

Schultz M, Chau W, Graham SJ, McIntosh AR, Ross B, Ishii R, Pantev C (2004) An integrative MEG-fMRI study of the primary somatosensory cortex using crossmodal correspondence analysis. NeuroImage 22:120-133.

Sederberg PB, Kahana MJ, Howard MW, Donner EJ, Madsen JR (2003) Theta and gamma oscillations during encoding predict subsequent recall. J Neurosci 23:10809-10814.

Singh KD, Barnes GR, Hillebrand A, Forde EM, Williams AL (2002) Taskrelated changes in cortical synchronization are spatially coincident with the hemodynamic response. NeuroImage 16:103-114.

Skaggs WE, McNaughton BL, Wilson MA, Barnes C (1996) Theta phase precession in hippocampal neuronal populations and the compression of temporal sequences. Hippocampus 6:149-172.

Spiers HJ, Burgess N, Maguire EA, Baxendale SA, Hartley T, Thompson P, O'Keefe J (2001) Unilateral temporal lobectomy patients show lateralized topographical and episodic memory deficits in a virtual town. Brain 124:2476-2489.

Squire LR, Stark CEL, Clark RE (2004) The medial temporal lobe. Annu Rev Neurosci 27:279-306.

Stockwell RG, Mansinha L, Lowe RP (1996) Localization of the complex spectrum: the S transform. IEEE Trans Signal Process 44:998-1000.

Tesche CD, Karhu J (2000) Theta oscillations index human hippocampal activation during a working memory task. Proc Natl Acad Sci USA 97:919-924.

Vinogradova OS (2001) Hippocampus as comparator: role of the two input and two output systems of the hippocampus in selection and registration of information. Hippocampus 11:578-598.

Vrba J, Robinson SE (1999) Signal processing in magnetoencephalography. Methods 25:249-271.

Wolbers T, Buchel C (2005) Dissociable retrospenial and hippocampal contributions to successful formation of survey representations. J Neurosci 25:3333-3340. 